\title{
Particle Imaging and Flow Visualization of In-situ TEM Nanoreactors
}

\author{
I. Puspitasari ${ }^{1}$, P. Saputra ${ }^{2}$, B. Morana ${ }^{3}$, L. Mele ${ }^{3}$, F. Santagata ${ }^{3}$, J.F. Creemer ${ }^{3}$, F. Kapteijn*, \\ P.J Kooyman ${ }^{1}$ \\ 1. ChemE, Applied Sciences, Delft University of Technology \\ 2. Precision and Microsystems Engineering, 3ME, Delft University of Technology \\ 3. DIMES-ECTM, Delft University of Technology
}

Knowledge about the mechanism of the catalytic reaction is important to achieve optimum performance of the catalyst. In-situ TEM is one method to study this mechanism. The catalytic reaction is performed inside the TEM using a nanoreactor which is very thin and transparent enough to enables the live imaging of the reaction. In the development of in-situ TEM different type of nanoreactors has been produced based on MEMS technology [1-3].

Loading the nano particles into a nanoreactor can be problematic due to surface charge, uniformity of particle size distribution and the design of the nanoreactor. In order to study the behavior of particle motion in the nanoreactor, a qualitative flow visualization of colloidal polystyrene suspension in the nanoreactor was analyzed. Three different particle sizes were tested: $0.2,0.5$ and $1 \mu \mathrm{m}$. A short wave length light source (i.e., mercury lamp) was used to illuminate a suspension of fluorescent PS particles. The particle motion was visualized and recorded using an inverted Zeiss Axiovert 200 fluorescence microscope, a CCD camera and processed using DAVIS imaging software. The loading method was to put one drop of suspension in the inlet, then let the capillary forces suck the suspension into the nanoreactor channel.

In this study we used two types of nanoreactor. The first nanoreactor was wafer bonded nanoreactor [2]. The nanoreactor was fabricated based on silicon fusion bonding and thin film encapsulation for sealed lateral electrical feedthroughs. The schematic cross section of the device is shown in Figure 1. The device had a narrow channel of $2 \mu \mathrm{m}$ height which enabled the particles up to $1 \mu \mathrm{m}$ particle size to enter. During the loading some bubbles formed on the heating area. The disappeared with time as the flow of the suspension continues. The dried nanoreactor showed that many particles were present on the heating area.

The second nanoreactor was fabricated by surface micromachining [3]. The 3D sketch of the device is shown in Figure 2. The device had a shallow channel of $0.5 \mu \mathrm{m}$. To increase the stiffness and prevent bulging, the top and bottom parts of the device are held by a line of pillars with the spacing of $20 \mu \mathrm{m}$ along the channel. During the fabrication, some holes were formed on the SiNx layer for sacrificial etching, to form the channel, and to do internal coating of the channel. At the end of the fabrication, the holes were plugged by PECVD SiNx. The design of these plugs caused some particles to be trapped inside during loading. The trapped particles and crowded pillars along the channel caused a heavy traffic jam in the inlet. Only low concentration suspensions could flow into the channel. When the solvent evaporated, it also swept away some particles then flowed into the outlet/inlet holes, leaving only a small amount of particles on the heating area.

We conclude that the geometry and the design of the nanoreactor affect the flow of suspension during loading. Single drop loading via the inlet leads to irregular distribution following evaporation 
of the liquid of the suspension. Double loading via both inlet and outlet seems to be effective to load particles onto the central area of the nanoreactor.

\section{References}

[1] J.F Creemer et al., Ultramicroscopy 108 (2008) 993.

[2] J.F Creemer et al., Proceedings of the IEEE International Conference on Micro Electro Mechanical System (MEMS) art. No. 5734622 (2011) 1103.

[3] L. Mele et al., Journal of Micromechanics and Microengineering 20 (2010) 085040.

[4] This research was supported by NIMIC (Nano Imaging under Industrial Condition), a SmartMix project of the Ministry of Economic Affair, The Netherlands.
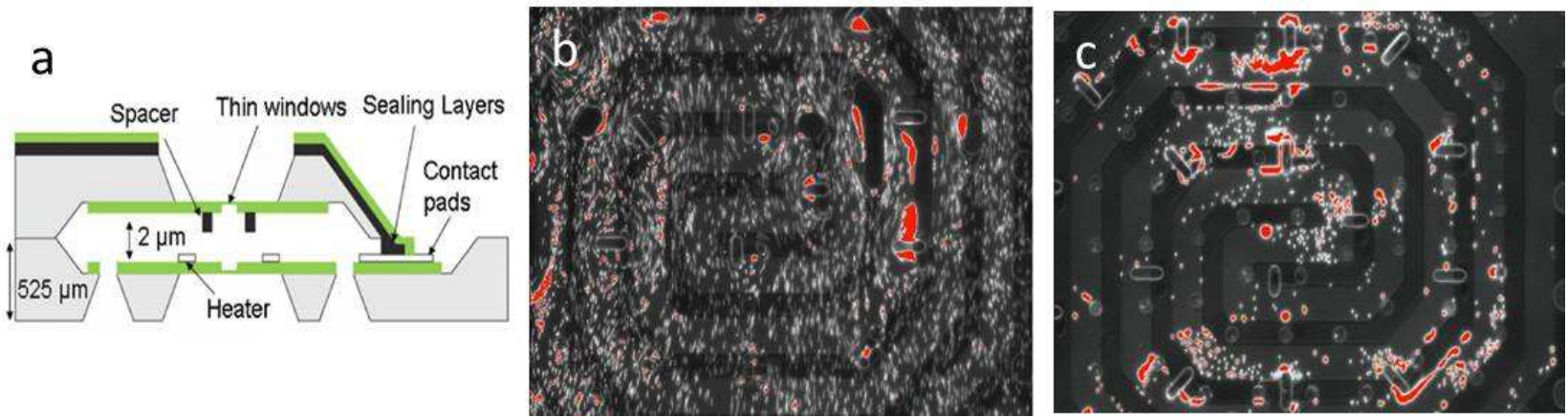

Figure 1. Schematic cross section of wafer bonded nanoreactor (a), bubble formation on the heating area during loading (b) and dried nanoreactor with some particles sit on the heating area (c).

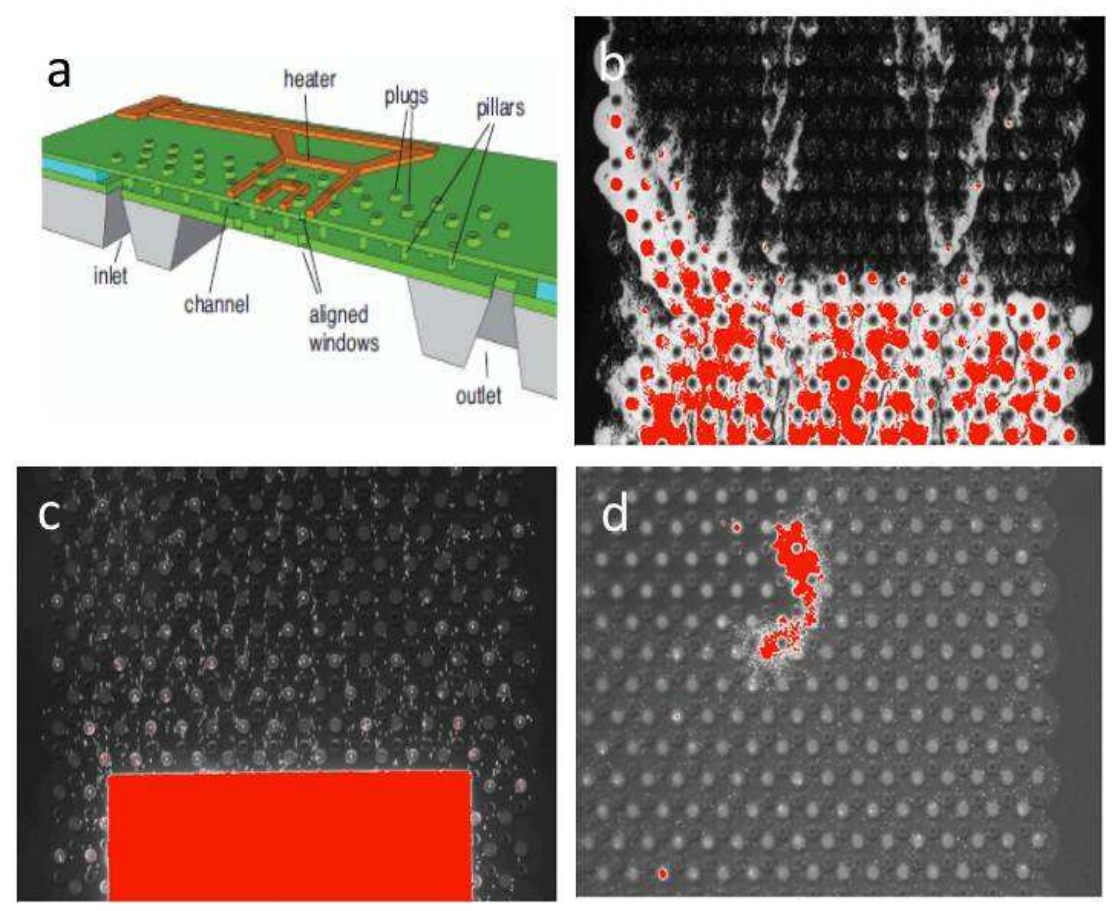

Figure 2. 3D sketch of surface micromachined nanoreactor (a), heavy traffic jam at the inlet during loading using high concentration suspension (b), streamline flow at the inlet during loading using low concentration suspension (c) and the evaporation process (d). 